\title{
Aspartate Aminotransferase Increased, CTCAE
}

National Cancer Institute

\section{Source}

National Cancer Institute. Aspartate Aminotransferase Increased, CT CAE. NCI

Thesaurus. Code C143301.

A finding based on laboratory test results that indicate an increase in the level of aspartate aminotransferase (AST or SGOT) in a blood specimen. 